\title{
Hospitalization rates and outcome of invasive bacterial vaccine-preventable diseases in Tuscany: a historical cohort study of the 2000-2016 period
}

Elena Chiappini ${ }^{1}$, Federica Inturrisi ${ }^{2}$, Elisa Orlandini ${ }^{3}$, Maurizio de Martino ${ }^{1}$ and Chiara de Waure ${ }^{4^{*}}$ (D)

\begin{abstract}
Background: Invasive bacterial diseases (IBD) are a serious cause of hospitalization, sequelae and mortality. Albeit a low incidence, an increase in cases due to $\mathrm{H}$. influenzae was registered in the past 4 years and, in the Tuscany region, an excess of cases due to $N$. meningitidis since 2015 is alarming. The purpose of this study is to deepen the knowledge of IBD epidemiology in Tuscany with particular attention to temporal trends.

Methods: Tuscan residents hospitalized for IBD from January 1st 2000 to March 18th 2016 were selected from the regional hospital discharge database based on ICD-9-CM codes. Age-specific and standardized hospitalization rates were calculated together with case-fatality rates (CFRs). A time-trend analysis was performed; whereas, prognostic factors of death were investigated through univariable and multivariable analyses.

Results: The average standardized hospitalization rates for invasive meningococcal diseases (IMD), invasive pneumococcal diseases and invasive diseases due to $H$. influenzae from 2000 to 2016 were $0.6,1.8$, and 0.2 per 100,000 , respectively. The average CFRs were $10.5 \%, 14.5 \%$ and $11.5 \%$ respectively with higher values in the elderly. Older age was significantly associated with higher risk of death from all IBD. A significant reduction in hospitalization rates for IMD was observed after meningococcal $C$ conjugate vaccine introduction. The Annual Percentage Change (APC) was -13.5 (95\% confidence interval (Cl) -22.3; -3.5) in 2005-2013 but has risen since that period. Furthermore, a significant increasing trend of invasive diseases due to $\mathrm{H}$. influenzae was observed from 2005 onwards in children 1-4 years old (APC 13.3; 95\% Cl 0; 28.3).

Conclusions: This study confirms changes in the epidemiology of invasive diseases due to $\mathrm{H}$. influenzae and IMD. Furthermore, attention is called to the prevention of IBD in the elderly because of the age group's significantly higher rate of hospitalizations and deaths for all types of IBD.
\end{abstract}

Keywords: Invasive bacterial diseases, Hospitalization, trend

\section{Background}

Invasive bacterial diseases (IBD) are an important public health issue and cause a serious burden in several countries, particularly among young persons and the elderly. The most common IBD clinical manifestations are septicemia and meningitis, with the first occurring even without the presence of the second, accounting together for

\footnotetext{
* Correspondence: chiara.dewaure@unipg.it

${ }^{4}$ Department of Experimental Medicine, University of Perugia, Piazzale

Gambuli 1, 06132 Perugia, Italy

Full list of author information is available at the end of the article
}

170,000 annual deaths worldwide [1, 2]. Meningitis is a severe infection of the meninges and can rapidly progress from the sudden onset of non-specific symptoms (including fever, nausea, vomiting, and neck stiffness) to death in 24 h. As many as $20-50 \%$ of the survivors may have permanent sequelae, such as hearing loss, amputation, or neurological and behavioral impairments [3-5]. Septicemia is a life-threatening condition that can cause tissue damage, organ failure, and death [6]. The three most common etiological agents of IBD are Haemophilus influenzae, Streptococcus pneumoniae, and Neisseria

(C) The Author(s). 2018 Open Access This article is distributed under the terms of the Creative Commons Attribution 4.0 International License (http://creativecommons.org/licenses/by/4.0/), which permits unrestricted use, distribution, and 
meningitidis. They are carried asymptomatically in the human nasopharynx and transmitted by aerosol droplets or secretions during close or lengthy contact. $H$. influenzae may be non-encapsulated (non-typeable) or encapsulated with a polysaccharide capsule. In the latter case, six serotypes (a-f) are recognized, with $H$. influenzae serotype b (Hib) being the most pathogenic. Before the availability of Hib conjugate vaccines in the late 1990s, $H$. influenzae was causing the majority of bacterial meningitis and IBD, while it now accounts for only $2-7 \%$ of cases [7-10]. Invasive diseases due to $H$. influenzae are most common in children below 5 years of age and rare in adolescents and adults [11]. With the decline of cases due to $H$. influenzae, S. pneumoniae became the leading cause of IBD, especially among children younger than 5 years of age, elderly and people with chronic diseases or immuno-compromised [12]. Out of 93 known S. pneumoniae serotypes, only 2030 are responsible for the majority of invasive pneumococcal diseases (IPD) worldwide [13]. Furthermore, $S$. pneumoniae is known as the leading cause of community-acquired pneumonia, and the lethality associated with bacteriaemic pneumococcal pneumonia is 6$20 \%$ [14]. The 7-valent pneumococcal conjugate vaccine (PCV7) for infants and young children was licensed in Europe in 2001, leading to a reduction in hospitalization rates for all S. pneumoniae -related diseases in children [15-17]. With the replacement of PCV7 with the 13-valent pneumococcal conjugate vaccine (PCV13) in 2010-2011 and the extension of vaccination to elderly and people at risk [18], it is likely that $N$. meningitidis will become a major agent of IBD worldwide, in particular if vaccination coverage will not reach high levels. Moreover, $N$. meningitidis is the only bacterium able to produce epidemics of meningitis. Incidence rates of invasive meningococcal diseases (IMD) are generally highest in children below 5 years of age followed by adolescents and young adults. Thirteen serogroups of $N$. meningitidis can be identified on the basis of the polysaccharide capsule but only six are responsible for most IMD cases: A, B, C, W135, X, and Y [2]. The distribution of these serogroups varies geographically, likely because of differences in population immunity and environmental factors. In Europe, serogroup B (MenB) is the main cause of IMD, accounting for up to $80 \%$ of cases in some countries, followed by serogroups C (MenC) and Y (MenY) $[2,19]$. With the introduction of the MenC conjugate vaccine (MCC) in the immunization schedules of several European countries, a significant decline in the incidence of MenC diseases has occurred over the past 10 years [19-21].

Nowadays, in Italy, PCV13 is given, together with Hib vaccine, in three doses within the first year of age $(3,5-$ 6, 11-13 months of age) [22]; whereas MCC is delivered in a single dose at $13-15$ months of age with a catch-up at 12-14 years of age [23]. In 2014-2015, a multicomponent MenB vaccine (4CMenB) has been introduced and is currently given in two, three or four doses depending on child's age at the time of vaccination. Nevertheless, until the launch of the new National Immunization Plan in January 2017, MenB vaccine has been offered free of charge only in few Italian regions [23-25]. Incidence rates for IBD are relatively low in all Italian regions. In 2014, national incidence rates were as follows: 0.17 per 100,000 for invasive $H$. influenzae diseases, 0.27 per 100,000 for IMD, and 1.57 per 100,000 for IPD. However, an increase in the incidence rates of invasive $H$. influenzae diseases was registered from 2011 [26]. Moreover, in particular in the Tuscany region, an excess of IMD cases was registered from January 2015 onwards [27]. Health authorities responded to the increasing number of cases with an extraordinary vaccination campaign $[28,29]$.

The present study aims to study the epidemiology of IBD in Tuscany, with particular attention to temporal trends. For the three types of IBD, over a 16-year period, we estimated: i) age-specific and standardized hospitalization rates and their relationship with vaccination coverage; ii) agespecific case-fatality rates (CFR); and iii) time-trends of age-specific hospitalization rates. Additionally, prognostic factors for death were investigated.

\section{Methods}

\section{Study design, case definition and data collection}

This is a historical cohort study conducted in Tuscany, an Italian region with a population of 3.7 million people. The regional hospital discharge database was accessed in order to identify patients admitted with a diagnosis of IBD due to $N$. meningitidis, S. pneumoniae or $H$. influenzae from January 1st 2000 to March 18th 2016. The database relies on the International Classification of Disease, Ninth Revision, Clinical Modification (ICD-9-CM) system that is currently used in Italy [30]. The project has been approved by the Ethics Committee of the "Azienda OspedalieroUniversitaria Meyer" of Florence on October 4th 2010 (authorization number 2010/7880).

Eligible patients were retrospectively searched in the regional hospital discharge database using the following ICD-9-CM codes for primary or secondary diagnosis:

- IMD: 036.0 (meningococcal meningitis), 036.1 (meningococcal encephalopathy), 036.2 (meningococcal septicemia), 036.40 (unspecified meningococcal carditis), 036.41 (meningococcal pericarditis), 036.42 (meningococcal endocarditis), 036.43 (meningococcal myocarditis), 036.81 (meningococcal optic neuritis), 036.82 (meningococcal arthropathy), 036.89 (other specified forms of meningococcal infection), and 036.9 (unspecified meningococcal infection); 
- IPD: 038.2 (pneumococcal septicemia), and 320.1 (pneumococcal meningitis);

- invasive H. influenzae diseases: 038.41 (septicemia due to H. influenzae), and 320.0 (Haemophilus meningitis).

Hospital discharge records of eligible patients included the following data: details of the admitting hospital, age, gender, nationality, region of residence, date of admission, one primary and five secondary diagnoses, surgical and other procedures, date and type of discharge. All hospitalized patients living in Tuscany and discharged from a Tuscan hospital, with one of the ICD-9-CM codes described above in primary or secondary diagnosis, were included in the study. One day-hospitalizations were excluded. A cross-check of data included in the hospital discharge record of included patients was conducted to avoid duplicates due to patients being transferred from a hospital to another one. With respect to the population at risk, the number of people residing in Tuscany during the study period (at January 1 st of each year), stratified by age and gender, was taken from the Italian National Statistical Institute (ISTAT) database [31]. Data on vaccination coverage of $H$. influenzae (Hib), S. pneumoniae (PCV) and $N$. meningitidis (MCC) at 24 months of age were searched on the Italian Ministry of Health database and ICONA studies [32-34].

\section{Statistical analysis}

Continuous variables were summarized using mean \pm standard deviation (SD), whereas categorical variables were reported as absolute and relative frequencies. Statistical analyses were carried out using STATA software version 13.1 except as otherwise specified.

\section{Descriptive analysis}

Age-specific hospitalization rates (HR), stratified for type of invasive disease, were calculated as cases per 100,000 together with 95\% confidence intervals (95\% CI). Resident population at January 1st of each year was used for the calculation. Standardized hospitalization rates (SHR) and their 95\% CI were considered to compare hospitalizations between years. Standardization was performed with respect to age and gender using the Italian population (latest available data from 2016) as external weight. $95 \%$ CIs were obtained as standardized rate \pm 1.96 *standard error (SE), and SE was calculated with the following Armitage and Berry formula [35]:

$$
\sqrt{\frac{\sum \frac{\left(T_{i} x N_{i}^{2} x K\right)}{n_{i}}}{\left(\sum N_{i}\right)^{2}}}
$$

$T_{i}=$ crude rate for each age class
$\mathrm{N}_{\mathrm{i}}$ = size of the reference population in each age class

$\mathrm{n}_{\mathrm{i}}=$ size of the study population in each age class

$\mathrm{K}=$ multiplication factor $(100,000)$.

Patients were stratified into seven age groups following the classification adopted by the National Surveillance System: <1, 1-4, 5-9, 10-14, 15-24, 25-64, $\geq 65$ years of age. Case-fatality rates (CFR) were calculated dividing the number of deaths by the number of cases and were presented stratified for type of invasive disease.

\section{Time-trend analysis}

Changes in overall and age-specific HRs from 2000 to 2015 were assessed by joinpoint (JP) regression according to Kim's method [36]. Data regarding 2016 were excluded from this analysis because they were partial. A joinpoint represents the time point when a significant trend change is detected. Time changes were expressed in terms of Annual Percent Change (APC) with 95\% CI. The null hypothesis was tested using a maximum of three changes in the slope with an overall significance level of 0.05 divided by the number of joinpoints in the final model. Joinpoint Regression program version 4.3.1 was used to carry out the analysis.

\section{Health outcome analysis}

Chi-square test was used to assess the relationships between the health outcome (dead, alive) and independent variables such as: age $(<5,5-17,18-64, \geq 65$ years of age), gender, nationality (Italian, non-Italian) and Charlson Index [37]. The Charlson Index was used as a proxy of comorbidity and calculated according to the algorithms developed by Quan et al. [38] looking at Enhanced ICD-9-CM Coding in primary and secondary diagnoses. The STATA additional software package "charlson" was used for this calculation. Variables with $p$-values below 0.25 at the univariable analysis were entered in a logistic regression model. The results were shown in terms of Odds Ratio (OR) and 95\% CI. All the analyses and models were carried out stratifying by type of invasive disease.

\section{Results \\ Descriptive analysis}

A total of 1691 patients with IBD were hospitalized between January 1st 2000 and March 18th 2016 at 52 hospitals in Tuscany region; 107 were residents outside the region and excluded from further analysis. Among the residents, 288 were children and adolescents ( $<18$ years), and 1296 were adults and elderly ( $\geq 18$ years). Additional file 1: Table S1 summarizes the study population's characteristics. Most children and adolescents were admitted to the Anna Meyer Children's University Hospital in Florence $(n=127 ; 44.1 \%)$. More than half of the hospitalizations of children and adolescents were due to IMD $(n=153 ; 53.1 \%)$ followed by IPD $(n=112$; 
$38.9 \%)$. Children's and adolescents' mean age was $4.8 \pm$ 5.2 years and the mean hospitalization length was $16 \pm$ 15.4 days; $56.9 \%$ of them were males and $92 \%$ were Italian. Among adults and elderly, IPD was the most common $\operatorname{IBD}(n=1017 ; 78.5 \%)$. Their mean age was $61.3 \pm 18$ years, males and females were equally represented and the majority was Italian. The mean length of hospitalization was $12 \pm 8.9$ days. Invasive $H$. influenzae diseases accounted for approximately $8 \%$ of cases in both groups. Data on vaccination coverage at 24 months of age in Tuscany were available for Hib from 2003 to 2015. Hib average coverage during the study period was of $94 \%$ but two drops to $88 \%$ were registered in 2003 and 2010. On the contrary, data for PCV and MCC vaccination coverage were retrieved only for 2003, 2008 and 2013-2015. Vaccination coverage was very low in 2003 and 2008, whereas it was between 92.9 and $94 \%$ for PCV and between 87.8 and $90.9 \%$ for MCC in 2013-2015.

More than one-quarter $(n=86 ; 26.1 \%)$ of the 330 hospitalizations for IMD occurred in children less than 4 years of age. No child $<1$ year of age died, while five died in the 1-4 years age group, yielding a CFR of $8.2 \%$ in this age group. People from 15 to 24 and from 25 to 64 years of age accounted for almost half $(n=158$; 47.9\%) of IMD hospitalizations, with CFRs of $8.0 \%$ and 9.6\% respectively. The highest number of deaths (11 deaths out of 42 cases) was registered in elderly ( $\geq 65$ years of age) with a CFR of $26.2 \%$. More than half $(n=581 ; 51.5 \%)$ of the 1129 hospitalizations for IPD occurred in people $\geq 65$ years of age with more than three-quarters $(n=122 ; 75.3 \%)$ of all deaths due to this infection, yielding a CFR of $21 \%$. Similarly, almost half ( $n=61 ; 48.8 \%)$ of the 125 hospitalizations for invasive $H$. influenzae diseases occurred in the $\geq 65$ years age group. All deaths for this IBD were registered in this age group (CFR: 21.3\%) except one in the 1-4 years age group (CFR: $11.1 \%)$ (Table 1). During the whole study period, CFR due to IMD had the lowest mean value (10.5\%, min
$0.0 \%, \max 33.3 \%)$. IPD and invasive $H$. influenzae diseases presented a mean CFR of $14.5 \%$ ( $\min 7.8 \%$, max $23.8 \%$ ) and $11.5 \%$ (min $0.0 \%$, max $50.0 \%$ ) (Fig. 1). Table 2 reports SHRs for type of IBD and year. The mean SHR for IMD was 0.6 per 100,000, with two peaks around 1 per 100,000 in 2004-2005 and in 2015. IPD had a mean SHR of 1.8 per 100,000 , ranging from 1.4 per 100,000 in 2010 to 2.2 per 100,000 in 2012. During the study period, invasive $H$. influenzae diseases had SHRs always below or equal to 0.3 per 100,000 (mean SHR: 0.2 per 100,000), with higher values in 2000-2005, 2012 and 2014 (Table 2). In the latter, the age-specific HRs for children $<1$ year of age and from 1 to 4 years of age showed peaks up to 7 per 100,000 children. The relationship between data on vaccination coverage and age-specific HRs for invasive $H$. influenzae diseases is shown in Fig. 2. On the contrary, the relationship between $\mathrm{PCV}$ and $\mathrm{MCC}$ vaccination coverage and HRs was not shown because of lack of data.

\section{Time-trend analysis}

The joinpoint analysis by type of IBD and age group is shown in Tables 3, 4 and 5. As far as IMD is concerned, considering all ages, joinpoints were found in 2005 and 2013 showing a significant decreasing trend in 20052013 (APC -13.4; 95\% CI -22.3; -3.5; $p<0.0001$ ) and a positive but non-significant trend before and after this time. No joinpoints or significant trends were shown for children up to 9 years old. However, while infants $<1$ year of age had a positive APC (APC 3.1; 95\% CI -5.1; 12.1), HRs for the $1-4$ years and $5-9$ years age groups tended to decrease (APC -5.9 and -7.6 respectively). The greatest reduction was found in the 10-14 years age group, whose APC decreased from 7.6 (95\% CI $-27.7 ; 60.2)$ in $2000-2004$ to -8.5 (95\% CI $-15.9 ;-0.4 ; p<0.0001)$ afterwards. No joinpoints were found in the other age groups. With respect to IPD, the overall trend was stable over time (APC 0.7; 95\% CI -0.6; 2.1). Infants $<1$ year of

Table 1 Absolute and relative frequencies of cases and deaths, and CFRs by age group due to IBD in Tuscany from 2000 to $2016^{\text {a }}$ $(N=1584)$

\begin{tabular}{|c|c|c|c|c|c|c|c|c|c|}
\hline \multirow[t]{2}{*}{ Age group } & \multicolumn{3}{|l|}{ IMD } & \multicolumn{3}{|l|}{ IPD } & \multicolumn{3}{|c|}{ Invasive $H$. influenzae diseases } \\
\hline & Cases n (\%) & Deaths n (\%) & CFR & Cases n (\%) & Deaths n (\%) & CFR & Cases n (\%) & Deaths n (\%) & CFR \\
\hline$<1$ year & $25(7.6)$ & $0(0.0)$ & 0.0 & $29(2.6)$ & $0(0.0)$ & 0.0 & $7(5.6)$ & $0(0.0)$ & 0.0 \\
\hline $1-4$ years & $61(18.5)$ & $5(16.1)$ & 8.2 & $52(4.6)$ & $0(0.0)$ & 0.0 & $9(7.2)$ & $1(7.1)$ & 11.1 \\
\hline $5-9$ years & $22(6.7)$ & $1(3.2)$ & 4.5 & $22(2.0)$ & $0(0.0)$ & 0.0 & $4(3.2)$ & $0(0.0)$ & 0.0 \\
\hline 10-14 years & $22(6.7)$ & $0(0.0)$ & 0.0 & $5(0.4)$ & $1(0.6)$ & 20.0 & $2(1.6)$ & $0(0.0)$ & 0.0 \\
\hline $15-24$ years & $75(22.7)$ & $6(19.4)$ & 8.0 & $14(1.2)$ & $1(0.6)$ & 7.1 & $2(1.6)$ & $0(0.0)$ & 0.0 \\
\hline $25-64$ years & $83(25.1)$ & $8(25.8)$ & 9.6 & $426(37.7)$ & $38(23.5)$ & 8.9 & $40(32.0)$ & $0(0.0)$ & 0.0 \\
\hline$\geq 65$ years & $42(12.7)$ & 11 (35.5) & 26.2 & $581(51.5)$ & $122(75.3)$ & 21.0 & $61(48.8)$ & $13(92.9)$ & 21.3 \\
\hline All ages & $330(100.0)$ & $31(100.0)$ & 9.4 & $1129(100.0)$ & $162(100.0)$ & 14.3 & $125(100.0)$ & $14(100.0)$ & 11.2 \\
\hline
\end{tabular}

aData refer up to March 2016

IMD invasive meningococcal diseases, IPD invasive pneumococcal diseases, CFR case-fatality rate 


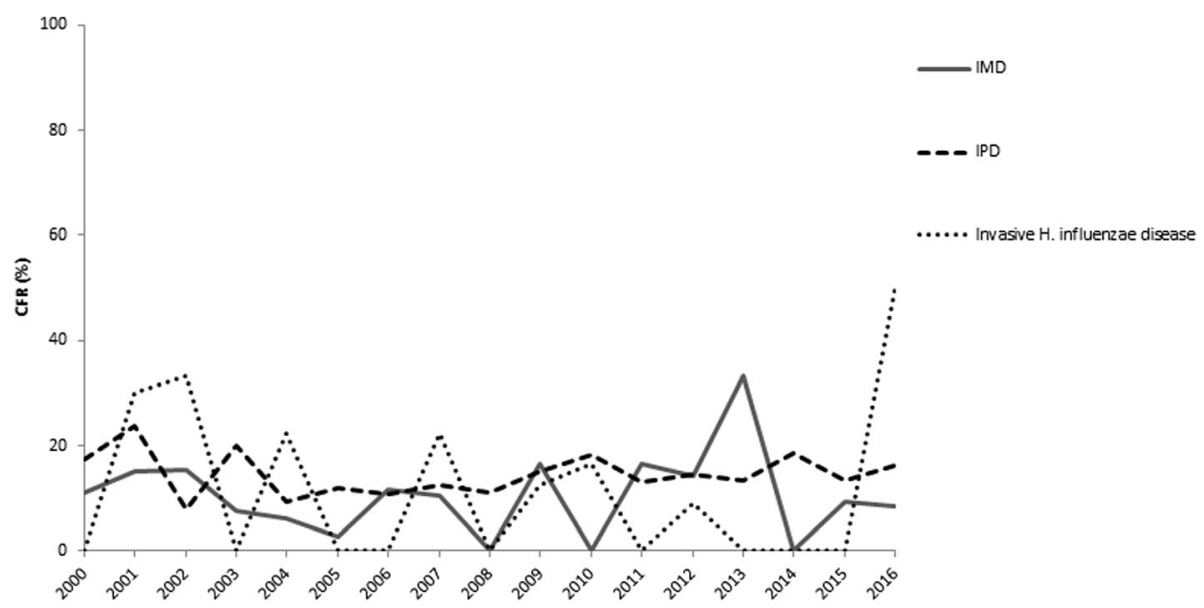

Fig. 1 Case-fatality rates (CFRs) per type of IBD from 2000 to 2016

age had an overall significant decreasing trend (APC -9.4; 95\% CI $-16 ;-2.3 ; p<0.0001$ ) (data not shown) with a joinpoint in 2004 and an APC of -16.7 in 2004-2015 (95\% CI $-28 ;-3.6 ; p<0.0001)$. Children from 1 to 4 years of age also had a negative but non-significant APC (APC -7; 95\% CI -13.7; 0.2). Joinpoints were found in the 10-14 years age group in 2002, 2009 and 2012, with positive and negative fluctuating trends. Young adults in the 15-24 years age group showed a joinpoint in 2003: APC changed from $95.2(95 \%$ CI $-50.5 ; 670.3)$ in

Table 2 Summary table of HRs (per 100,000) standardized for age and gender

\begin{tabular}{llll}
\hline Years & IMD & IPD & Invasive H. influenzae diseases \\
\hline 2000 & 0.552 & 1.505 & 0.246 \\
2001 & 0.593 & 1.789 & 0.307 \\
2002 & 0.822 & 1.808 & 0.252 \\
2003 & 0.373 & 2.057 & 0.329 \\
2004 & 0.953 & 1.780 & 0.285 \\
2005 & 1.067 & 2.046 & 0.217 \\
2006 & 0.486 & 1.799 & 0.162 \\
2007 & 0.557 & 1.920 & 0.251 \\
2008 & 0.488 & 1.896 & 0.063 \\
2009 & 0.508 & 1.748 & 0.202 \\
2010 & 0.437 & 1.440 & 0.158 \\
2011 & 0.352 & 2.002 & 0.084 \\
2012 & 0.381 & 2.170 & 0.281 \\
2013 & 0.250 & 1.741 & 0.109 \\
2014 & 0.492 & 1.621 & 0.279 \\
2015 & 0.904 & 2.048 & 0.205 \\
$2016^{\mathrm{a}}$ & 0.336 & 0.783 & 0.051 \\
\hline
\end{tabular}

${ }^{a}$ Data refer up to March 2016

$H R$ hospitalization rate, IMD invasive meningococcal diseases, IPD invasive pneumococcal diseases
2000-2003 to -13.4 (95\% CI $-22.5 ;-3.3 ; p<0.0001$ ) afterwards. No joinpoints were found in adults and elderly (positive non-significant trends). For invasive $H$. influenzae diseases, there was an overall decreasing but non-significant trend (APC -1.5; 95\% CI -5.3; 2.4). Joinpoints were found in all age groups except for adults and elderly. Infants $<1$ year of age had a significant decreasing trend in 2000-2008 (APC -17.5; 95\% CI $-30.5 ;-2.1 ; p<0.0001)$ followed by an increasing but non-significant trend. Joinpoints were found in children from 1 to 4 years of age in 2002 and 2005: a significant increase was seen in 2005-2015 (APC 13.3; 95\% CI 0.0; 28.3; $p<0.0001)$. On the contrary, the trend decreased for children 5-9 years old in 2008-2015 (APC -15.1; $95 \%$ CI $-24 ;-5.1 ; p<0.0001)$. In the $10-14$ years age group, joinpoints were found in 2003, 2009 and 2012, with positive and negative fluctuating trends. Young adults in the 15-24 years age group had an overall significant decreasing trend (APC -3.4; 95\% CI -5.5; -1.4; $p<0.0001$ ) (data not shown) with a joinpoint in 2003 and an APC of -19.3 in 2003-2015 (95\% CI -30.4; -6.5; $p<0.0001)$. An exemplary time-trend change is illustrated in Additional file 2: Figure S1.

\section{Health outcome analysis}

The univariable analyses showed that there were more deaths in older patients, in females, in Italian patients, and in patients with comorbidities. Among all deaths due to IMD, only $9(29 \%)$ were registered in the pediatric age as compared to $22(71 \%)$ in people $\geq 18$ years of age $(p=0.004)$. The same was observed for IPD $(p<0.0001)$ and invasive $H$. influenzae diseases $(p=0.003)$. Age was entered into all IBD logistic regression models and, in light of small absolute frequencies, it was classified as $<18$ years vs $\geq 18$ years of age for IPD and as $<65$ years vs $\geq 65$ years of age for invasive $H$. influenzae diseases. 


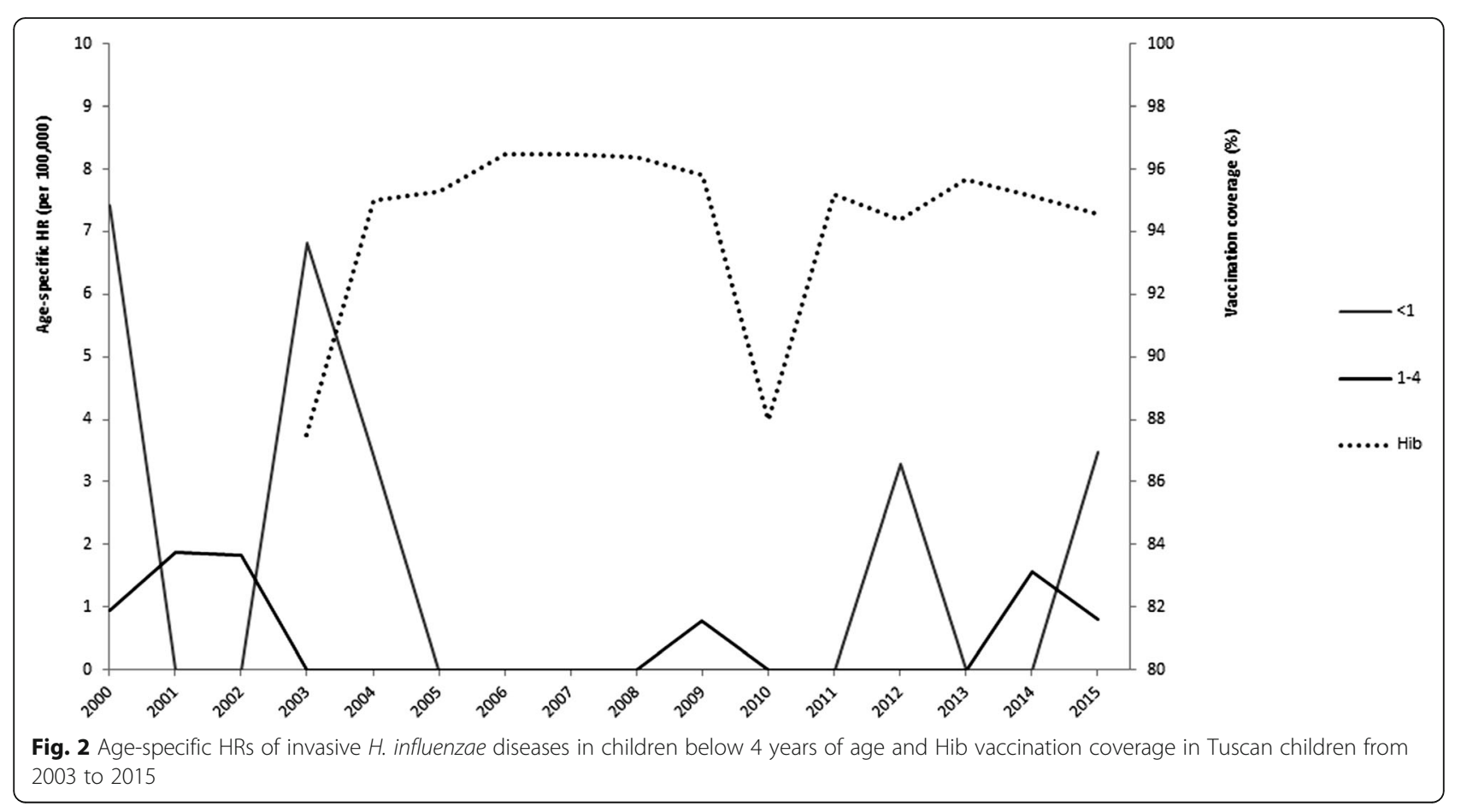

Gender was not shown to be significantly associated to death, but it was kept in the IMD and IPD logistic regression models. Among patients who died for IPD, there were more Italians than non-Italians $(p=0.052)$. Significant associations were not found for the other two IBD, therefore nationality was entered only into the logistic regression model for IPD. As for the Charlson Index, a smaller percentage of people without comorbidities was observed among patients who died as compared to those who survived. For instance, $90 \%$ of IMD patients discharged alive had no comorbidities in comparison to

Table 3 Findings of the joinpoint regression for IMD by age group

\begin{tabular}{lllll}
\hline Age group & Joinpoint & Years Range & APC $(95 \% \mathrm{Cl})$ & $p$-value \\
\hline All age groups & 2005 & $2000-2005$ & $9.9(-5.3 ; 27.5)$ & 0.2 \\
& 2013 & $2005-2013$ & $-13.4(-22.3 ;-3.5)$ & $<0.0001$ \\
& & $2013-2015$ & $80(-7.3 ; 249.5)$ & 0.1 \\
$<1$ year & No JP & $2000-2015$ & $3.1(-5.1 ; 12.1)$ & 0.4 \\
1-4 years & No JP & $2000-2015$ & $-5.9(-11.6 ; 0.2)$ & 0.1 \\
5-9 years & No JP & $2000-2015$ & $-7.6(-14.9 ; 0.4)$ & 0.1 \\
10-14 years & 2004 & $2000-2004$ & $7.6(-27.7 ; 60.2)$ & 0.7 \\
& & $2004-2015$ & $-8.5(-15.9 ;-0.4)$ & $<0.0001$ \\
15-24 years & No JP & $2000-2015$ & $0.2(-6.2 ; 7.2)$ & 0.9 \\
25-64 years & No JP & $2000-2015$ & $4.2(-2.9: 11.8)$ & 0.2 \\
$\geq 65$ years & No JP & $2000-2015$ & $-4.5(-12.3 ; 3.9)$ & 0.3 \\
\hline
\end{tabular}

$I M D$ invasive meningococcal diseases, $A P C$ annual percent change, $\mathrm{Cl}$ confidence interval, JP joinpoint
$74.2 \%$ among who died $(p=0.014)$. Significant associations were also seen in the other two IBD and Charlson Index was entered into all models as a dichotomous variable (presence/absence of comorbidities) (Table 6).

The final logistic regression models for type of invasive disease are shown in Table 7. They were overall statistically significant and demonstrated that older age was a risk factor for dying for all IBD. In particular, the IMD model was entirely explained by the variable age, with

Table 4 Findings of the joinpoint regression for IPD by age group

\begin{tabular}{lllll}
\hline Age group & Joinpoint & Years Range & APC $(95 \%$ Cl $)$ & $p$-value \\
\hline All age groups & No JP & $2000-2015$ & $0.7(-0.6 ; 2.1)$ & 0.3 \\
$<1$ year & 2004 & $2000-2004$ & $15.5(-25.5 ; 78.9)$ & 0.5 \\
& & $2004-2015$ & $-16.7(-28 ;-3.6)$ & $<0.0001$ \\
1-4 years & No JP & $2000-2015$ & $-7(-13.7 ; 0.2)$ & 0.1 \\
5-9 years & No JP & $2000-2015$ & $0.8(-9.5 ; 12.2)$ & 0.9 \\
10-14 years & 2002 & $2000-2002$ & $81.9(79.6 ; 84.3)$ & $<0.0001$ \\
& 2009 & $2002-2009$ & $-20.4(-20.6 ;-20.2)$ & $<0.0001$ \\
& 2012 & $2009-2012$ & $99(96.4 ; 101.6)$ & $<0.0001$ \\
& & $2012-2015$ & $-50.1(-50.4 ;-49.7)$ & $<0.0001$ \\
$15-24$ years & 2003 & $2000-2003$ & $95.2(-50.5 ; 670.3)$ & 0.3 \\
& & $2003-2015$ & $-13.4(-22.5 ;-3.3)$ & $<0.0001$ \\
25-64 years & No JP & $2000-2015$ & $0.8(-1.8 ; 3.5)$ & 0.5 \\
$\geq 65$ years & No JP & $2000-2015$ & $1.7(-0.5 ; 3.8)$ & 0.1 \\
\hline
\end{tabular}

IPD invasive pneumococcal diseases, $A P C$ annual percent change, $\mathrm{Cl}$ confidence interval, $J P$ joinpoint 
Table 5 Findings of the joinpoint regression for invasive $H$. influenzae diseases by age group

\begin{tabular}{lllll}
\hline Age group & Joinpoint & Years Range & APC $(95 \%$ CI $)$ & $p$-value \\
\hline All age groups & No JP & $2000-2015$ & $-1.5(-5.3 ; 2.4)$ & 0.4 \\
$<1$ year & 2008 & $2000-2008$ & $-17.5(-30.5 ;-2.1)$ & $<0.0001$ \\
& & $2008-2015$ & $10.8(-12.9 ; 40.9)$ & 0.4 \\
1-4 years & 2002 & $2000-2002$ & $21.5(-64.4 ; 314.2)$ & 0.7 \\
& 2005 & $2002-2005$ & $-44.4(-92.5 ; 312.1)$ & 0.5 \\
& & $2005-2015$ & $13.3(0.0 ; 28.3)$ & $<0.0001$ \\
5-9 years & 2008 & $2000-2008$ & $7.6(-1.3 ; 17.2)$ & 0.1 \\
& & $2008-2015$ & $-15.1(-24.0 ;-5.1)$ & $<0.0001$ \\
10-14 years & 2003 & $2000-2003$ & $17.2(16.5 ; 18.0)$ & $<0.0001$ \\
& 2009 & $2003-2009$ & $-9.8(-10.0 ;-9.5)$ & $<0.0001$ \\
& 2012 & $2009-2012$ & $23.4(21.9 ; 24.9)$ & $<0.0001$ \\
& & $2012-2015$ & $-20.6(-21.1 ;-20.1)$ & $<0.0001$ \\
$15-24$ years & 2003 & $2000-2003$ & $-19.3(-30.4 ;-6.5)$ & $<0.0001$ \\
& & $2003-2015$ & $-0.1(-2.1 ; 2.0)$ & 0.9 \\
25-64 years & No JP & $2000-2015$ & $-0.7(-7.9 ; 7.1)$ & 0.8 \\
$\geq 65$ years & No JP & $2000-2015$ & $-1.4(-7.1 ; 4.6)$ & 0.6 \\
\hline
\end{tabular}

$A P C$ annual percent change, $\mathrm{Cl}$ confidence interval, JP joinpoint

older age ( $\geq 65$ years old) associated to a higher risk of dying (OR 3.13; 95\% CI 1.14; 8.60) compared to adults (18-64 years old). As for the IPD model, adults and elderly (OR 17.43; 95\% CI 2.40; 126.35) and patients with comorbidities (OR 1.45; 95\% CI 1.03; 2.04) had a higher risk of death compared to patients in pediatric age and without comorbidities respectively. Similarly, to IMD, the model for invasive $H$. influenzae diseases was entirely explained by the variable age: elderly ( $\geq 65$ years of age) showed a higher risk of death (OR 17.94; $95 \%$ CI $2.16 ; 148.71)$.

\section{Discussion}

This historical observational study assessed the trends of IBD hospitalizations in a population of 3.7 million people over the past 16 years. The findings highlighted decreasing hospitalization rates for IPD in infants $<1$ year of age, likely because of the effects of PCV vaccines. A similar reduction in S. pneumoniae-related hospitalizations in children was shown in two other Italian regions, Friuli Venezia Giulia and Veneto [17]. Although data were limited, high PCV vaccination coverages retrieved for 20132015 support the conclusion. The data showed that, in the last few years, hospitalization rates for invasive $H$. influen$z a e$ diseases increased, as also reported by the National Surveillance System over the past 4 years. In fact, an increasing trend, although non-significant, was observed in infant $<1$ year of age from 2008 onwards, and a significantly increasing trend was shown in the 1-4 years age group from 2005 onwards. One can speculate that this increase was linked, among other reasons, to a drop in $\mathrm{Hib}$ vaccination coverage. Thanks to the high vaccination coverage reached in almost all Italian regions, cases attributable to serotype b, the only ones preventable by vaccination, are rare [26]. Nevertheless, the common belief that invasive $H$. influenzae diseases have disappeared after the introduction of the vaccine is not supported and should go no further. In fact, on average, one case out of nine per

Table 6 Findings of univariable analyses performed by chi-square test

\begin{tabular}{|c|c|c|c|c|c|c|c|c|c|}
\hline \multirow[t]{2}{*}{ Variable } & \multicolumn{3}{|l|}{$\mathrm{IMD}$} & \multicolumn{3}{|l|}{ IPD } & \multicolumn{3}{|c|}{ Invasive $H$. influenzae diseases } \\
\hline & Dead n (\%) & Alive n (\%) & $p$-value & Dead n (\%) & Alive n (\%) & $p$-value & Dead n (\%) & Alive n (\%) & $p$-value \\
\hline \multicolumn{10}{|l|}{ Age group } \\
\hline$<5$ years & $5(16.1)$ & $81(27.1)$ & 0.004 & $0(0.0)$ & $81(8.4)$ & $<0.0001$ & $1(7.1)$ & $15(13.5)$ & 0.003 \\
\hline 5-17 years & $4(12.9)$ & $63(21.1)$ & & $1(0.6)$ & $30(3.1)$ & & $0(0.0)$ & $7(6.3)$ & \\
\hline 18-64 years & $11(35.5)$ & $124(41.5)$ & & $39(24.1)$ & $397(41.1)$ & & $0(0.0)$ & $41(36.9)$ & \\
\hline$\geq 65$ years & $11(35.5)$ & $31(10.4)$ & & $122(75.3)$ & $459(47.4)$ & & $13(92.9)$ & $48(43.2)$ & \\
\hline \multicolumn{10}{|l|}{ Gender } \\
\hline male & $10(32.3)$ & $145(48.5)$ & 0.085 & $77(47.5)$ & $514(53.1)$ & 0.185 & $7(50.0)$ & $61(55.0)$ & 0.726 \\
\hline female & $21(67.7)$ & $154(51.5)$ & & $85(52.5)$ & $453(46.9)$ & & $7(50.0)$ & $50(45.0)$ & \\
\hline \multicolumn{10}{|l|}{ Nationality } \\
\hline Italian & $29(96.7)$ & $278(93.6)$ & 1.000 & $160(98.8)$ & $919(95.4)$ & 0.052 & $14(100.0)$ & 109 (98.2) & 1.000 \\
\hline non-Italian & $1(3.3)$ & $19(6.4)$ & & $2(1.2)$ & $44(4.6)$ & & $0(0.0)$ & $2(1.8)$ & \\
\hline \multicolumn{10}{|l|}{ Charlson Index } \\
\hline 0 & $23(74.2)$ & $269(90.0)$ & 0.014 & $88(54.3)$ & $643(66.5)$ & 0.004 & $6(42.9)$ & $64(57.7)$ & 0.090 \\
\hline 1 & 7 (22.6) & $19(6.3)$ & & $41(25.3)$ & $153(15.8)$ & & $1(7.1)$ & $22(19.8)$ & \\
\hline 2 & $1(3.2)$ & $11(6.7)$ & & $33(20.4)$ & $171(17.7)$ & & $7(50.0)$ & $25(22.5)$ & \\
\hline
\end{tabular}


Table 7 Findings of multivariable logistic regression models

\begin{tabular}{|c|c|c|c|}
\hline Type of IBD & Variable & OR $(95 \% \mathrm{Cl})$ & $p$-value \\
\hline \multirow[t]{11}{*}{$\mathrm{IMD}\left(N=330 ; p<0.05 ; R^{2}=0.07\right)$} & Age group & & \\
\hline & $<5$ years & $0.76(0.25 ; 2.30)$ & 0.624 \\
\hline & $5-17$ years & $0.79(0.24 ; 2.61)$ & 0.696 \\
\hline & 18-64 years & 1.00 & \\
\hline & $\geq 65$ years & $3.13(1.14 ; 8.60)$ & 0.027 \\
\hline & Gender & & \\
\hline & male & $0.70(0.30 ; 1.62)$ & 0.410 \\
\hline & female & 1.00 & \\
\hline & Charlson Index & & \\
\hline & no comorbidities & 1.00 & \\
\hline & comorbidities & $1.58(0.56 ; 4.42)$ & 0.385 \\
\hline \multirow[t]{12}{*}{$\operatorname{IPD}\left(N=1125 ; p<0.001 ; R^{2}=0.04\right)$} & Age group & & \\
\hline & $<18$ years & 1.00 & \\
\hline & $\geq 18$ years & $17.43(2.40 ; 126.35)$ & 0.005 \\
\hline & Gender & & \\
\hline & male & $0.81(0.58 ; 1.13)$ & 0.217 \\
\hline & female & 1.00 & \\
\hline & Nationality & & \\
\hline & Italian & $3.38(0.80 ; 14.20)$ & 0.097 \\
\hline & non-Italian & 1.00 & \\
\hline & Charlson Index & & \\
\hline & no comorbidities & 1.00 & \\
\hline & comorbidities & $1.45(1.03 ; 2.04)$ & 0.031 \\
\hline \multirow[t]{6}{*}{ Invasive $H$. influenzae diseases $\left(N=125 ; p<0.001 ; R^{2}=0.16\right)$} & Age group & & \\
\hline & $<65$ years & 1.00 & \\
\hline & $\geq 65$ years & $17.94(2.16 ; 148.71)$ & 0.007 \\
\hline & Charlson Index & & \\
\hline & no comorbidities & 1.00 & \\
\hline & comorbidities & $0.87(0.26 ; 2.92)$ & 0.821 \\
\hline
\end{tabular}

IBD invasive bacterial disease, $O R$ odds ratio, $C I$ confidence interval, IMD invasive meningococcal diseases, IPD invasive pneumococcal diseases

year $(11.1 \%)$ occurred in children $<4$ years of age in 2012 2015. Overall, HRs for IPD were in line with national estimates, whereas HRs for invasive H. influenzae diseases, although in line with estimates reported by some Italian regions, were higher than national estimates presumably affected by underreporting [26]. A more complex situation emerged for IMD, whose HRs appeared to be, on average, higher than the national ones $(0.6$ per 100,000 vs 0.3 per $100,000)$ [26], with two high peaks in 2004-2005 and in 2015. Considering that the time-trend analysis also revealed joinpoints around those years, we can assume two crucial changes in the epidemiology of IMD in Tuscany. The first likely reflects the introduction of MCC vaccine in 2005 with ensuing reduction of hospitalizations especially in children from 1 to 4 years of age (although non-significant) and in adolescents from
10 to 14 years of age, both primary targets of the vaccination campaign. These findings were also in line with a recent time-trend analysis investigating the impact of the MCC vaccine introduction in Italy [39]. Nevertheless, not enough data on vaccination coverage were available and thus no relationship could be determined in changes in age-specific HRs in relation to vaccination. The second change is in line with the increasing number of cases reported in young adults by the National Surveillance System in 2015 and in the first quarter of 2016 [26]. This change was also seen in our data (in particular regarding the 15-24 years age group) with nine and three cases respectively compared to one case per year in the previous 2 years (data not shown). This brought to the implementation of extraordinary measures and to vaccinate, free of charge, people 
between 20 and 45 years of age and, under request and with co-payment, people above 45 years of age $[28,29]$. It is important to note that, although no death occurred, 25 of the 330 IMD cases (7.6\%) affected infants $<1$ year and that the trend of HR for this age group was the only positive one in the pediatric age (although non-significant). In Tuscany, as well as in other Italian regions, infants $<1$ years of age are not covered by the $M C C$ vaccination that is offered at 13th months of age. The introduction of MenB vaccine for infants from 2014 onward can be expected to produce some benefits in years to come.

Regarding IBDs in adults, a note on IMD is warranted. Although the increased number observed in 2015-2016 has been mainly registered in young adults, adults from 25 to 64 years old contributed with half of the total cases per year: 16 cases in 2015 and six in the first quarter of 2016 as compared to three cases per year in the previous 2 years (data not shown). Time-trend analysis for this age group showed a positive but non-significant trend. Attention should be paid to this fast-changing situation also considering that adults above 45 years of age are not strictly a target of extraordinary $\mathrm{MCC}$ vaccination measures.

A deeper analysis is needed for the elderly. Despite the decreasing trend in IBD in vaccinated children (direct effect) and in unvaccinated subjects of all ages (indirect effect) [40-43], the level of disease control in the elderly is suboptimal. In fact, although no time-trends were observed, the absolute number of cases and CFRs remain high for all three IBD. For example, 42 of the 330 IMD cases (12.7\%) occurred in people $\geq 65$ years old, contributing with the highest number of deaths (11 out of 31 deaths due to IMD). This would appear even more relevant looking at invasive $H$. influenzae diseases: 61 out of the 125 cases (49\%) were registered in elderly as well as all deaths except one. The age-specific CFRs were higher than European estimates reported by the European Centre for Disease Prevention and Control (ECDC) [1]: $26.2 \%$ vs $17.1 \%$ for IMD, $21 \%$ vs $14.3 \%$ for IPD, and $21.3 \%$ vs $15 \%$ for invasive $H$. influenzae diseases. Furthermore, in all three regression models, older age was significantly associated with a higher risk for death. Several studies in the literature found older age and Charlson comorbidities to be independent predictors of death [44, 45]. This evidence calls for actions to extend out high vaccination coverage in elderly and people with chronic conditions to prevent the occurrence of such IBD, in particular IPD, in these groups.

In fact, while MCC and Hib vaccines are not given to the elderly, the 23-valent pneumococcal polysaccharide vaccine (PPV23) first and then the PCV13 have been used in Italy in people $\geq 65$ years of age. Nevertheless, albeit data on adults vaccination coverage are not routinely collected, local or regional studies suggest that vaccination coverage in people $\geq 65$ years of age is quite low, varying from 0.7 to $50 \%$ between 2004 and 2008 [22], when the PPV23 was administered to elderly [46]. A recently concluded randomized trial in the Netherlands provide the missing evidence of PCV13 efficacy in preventing vaccine-type IPD in older adults [47]. This evidence, together with an increased awareness of the problem of IBD in the elderly, should support policy makers in their decisions on the implementation of pneumococcal vaccination. This is envisaged also because vaccinating elderly against $S$. pneumoniae may prevent not only IPD but also pneumonia, which causes 1 million hospitalizations in Europe, costs about $€ 10$ billion per year, and represents the most frequent cause of death from infection $[48,49]$.

A positive point of the present study is that it gives a thorough overview of the epidemiology of IBD yielding also CFRs. This measure is widely used as an outcome indicator to make comparisons over time and between areas as its calculation is less prone to bias [50]. Moreover, compared to the National Surveillance System which included invasive diseases since 2007 only [22], this study was able to provide a picture of IBD over a wider time window overcoming the apparent increase in the number of cases which occurred in the National Surveillance System. Our study presents also some limitations. One is concerning the general sparse-cells problem that makes joinpoint models unstable and may justify fluctuating positive and negative trends in children from 10 to 14 years of age both for IPD and invasive $H$. influenzae diseases. Furthermore, it should be kept in mind that in presence of zero-counts, small absolute fluctuation may have a great relative impact. Another limitation was the lack of information on death after discharge from the hospital or transfer to a private non-accredited institute even though we consider it improbable to affect our results as the number of transferred patients was very low. Another limitation is represented by the lack of information on serotype distribution and individual vaccination records that could have allowed a more in-depth analysis of the relationship between vaccination and the occurrence of diseases. Additionally, over the past 16 years, diagnostics methods have become more sensitive and life support techniques could have influenced the health outcome. Finally, R-square values of regression models were very low, ranging between 4 and $16 \%$ because of the limited number of variables available from the hospital discharge records.

In conclusion, the results of our study contribute to the body of evidence on the epidemiology of IBD and the importance of ensuring high vaccination coverage. A constant effort should be made to attain and maintain high vaccination coverage among children in order to 
further reduce the incidence of all IBD and control apparent increasing trends. In particular, attention should be paid to the increase in invasive $H$. influenzae diseases and to the changing epidemiological scenario of IMD. Furthermore, actions should be also promoted to implement vaccination in the elderly. Eventually, prevention remains the most valuable tool to help reducing the burden of IBD in all age groups.

\section{Conclusion}

This study shows changes in the epidemiology of IBD, particularly due to $H$. influenzae and $N$. meningitidis, and high rates of hospitalizations and deaths for all types of IBD in elderly. This evidence calls for actions in order to maintain high vaccination coverage among children and promote vaccination in older age groups.

\section{Additional files}

Additional file 1: Table S1. Characteristics of patients with IBD resident in Tuscany in 2000-2016 ( $N=1584)$. (DOCX $12 \mathrm{~kb}$ )

Additional file 2: Figure S1. Joinpoint regression of IMD HRs, all ages, years 2000-2015. (TIF $61 \mathrm{~kb}$ )

\begin{abstract}
Abbreviations
4CMenB: multicomponent MenB vaccine; APC: Annual Percentage Change; CFR: case-fatality rates; Cl: confidence interval; ECDC: European Centre for Disease Prevention and Control; Hib: $\mathrm{H}$. influenzae serotype b; HR: hospitalization rates; IBD: invasive bacterial diseases; ICD-9CM: International Classification of Disease, Ninth Revision, Clinical Modification; IMD: invasive meningococcal diseases; IPD: invasive pneumococcal diseases; JP: joinpoint; MCC: MenC conjugate vaccine; MenB: meningococcal serogroup B; MenC: meningococcal serogroups C; MenY: meningococcal serogroup Y; OR: Odds Ratio; PCV13: 13-valent pneumococcal conjugate vaccine; PCV7: 7-valent pneumococcal conjugate vaccine; SE: standard error
\end{abstract}

\section{Availability of data and materials}

The dataset analysed during the current study is not publicly available but are available from the corresponding author on reasonable request.

\section{Authors' contributions}

EC, FI and CdW designed the study. EO collected the data, FI performed the statistical analysis and EC, CdW and MdM contributed to data interpretation. $\mathrm{Fl}$ and $\mathrm{CdW}$ drafted the manuscript and EC, EO and MdM critically revised it. All authors have read and approved the final manuscript and agreed to be accountable for all aspects of the work.

\section{Ethics approval and consent to participate}

The project has been approved by the Ethics Committee of the university hospital "Azienda Ospedaliero-Universitaria Meyer" of Florence on October 4th 2010 (authorization number 2010/7880). Data were obtained from an electronic database using ICD-9-CM. A specific informed consent was not considered necessary according to the Ethics Committee approving the project, because this was a descriptive epidemiological study performed on administrative data, which are routinely collected from any hospitalized patient after obtaining his/her consent. Furthermore, no human experimentation was foreseen by the study and patient information was anonymized and deidentified prior to analysis.

\section{Consent for publication}

Not applicable.

\section{Competing interests}

The authors declare that they have no competing interests. CdW is Associate Editor of BMC Health Services Research and BMC Infectious Diseases.

\section{Publisher's Note}

Springer Nature remains neutral with regard to jurisdictional claims in published maps and institutional affiliations.

\section{Author details}

${ }^{1}$ Anna Meyer Children's University Hospital, Department of Health Sciences, University of Florence, Florence, Italy. ${ }^{2}$ Department of Epidemiology \& Biostatistics, VU University Medical Center (VUmc), Amsterdam, the Netherlands. ${ }^{3}$ Tuscany Regional Government Department of Right to Health and Solidarity Policies, Information Technology Section, Florence, Italy. ${ }^{4}$ Department of Experimental Medicine, University of Perugia, Piazzale Gambuli 1, 06132 Perugia, Italy.

Received: 24 April 2017 Accepted: 6 August 2018

Published online: 13 August 2018

\section{References}

1. European Centre for Diseases Prevention and Control (ECDC). Surveillance of invasive bacterial diseases in Europe. Stockholm. 2011:2013. https://doi. org/10.2900/1510

2. World Health Organization (WHO). Meningococcal meningitis. Fact Sheet No141 2015. http://www.who.int/mediacentre/factsheets/fs141/en/. (Accessed 3 June 2016).

3. Rosenstein NE, Perkins BA, Stephens DS, Popovic T, Hughes JM. Meningococcal disease. N Engl J Med. 2001;344:1378-88. https://doi.org/10. 1056/NEJM200105033441807.

4. Stein-Zamir C, Shoob H, Sokolov I, Kunbar A, Abramson N, Zimmerman D. The clinical features and long-term sequelae of invasive meningococcal disease in children. Pediatr Infect Dis J. 2014;33:777-9. https://doi.org/10. 1097/INF.0000000000000282.

5. van de Beek D. Progress and challenges in bacterial meningitis. Lancet. 2012;380:1623-4. https://doi.org/10.1016/S0140-6736(12)61808-X.

6. Center for Disease Control and Prevention (CDC), World Health Organization (WHO). Epidemiology of Meningitis Caused by Neisseria meningitidis, Streptococcus pneumoniae, and Haemophilus influenzae. Lab. Methods Diagnosis Meningitis caused by Neisseria meningitidis, Streptococcus pneumoniae, Haemophilus Influ. 2nd ed., 2011.

7. Ladhani S, Slack MPE, Heath PT, von Gottberg A, Chandra M, Ramsay ME, et al. Invasive Haemophilus influenzae disease, Europe, 1996-2006. Emerg Infect Dis. 2010;16:455-63. https://doi.org/10.3201/eid1603.090290.

8. Schuchat A, Robinson K, Wenger JD, Harrison LH, Farley M, Reingold AL, et al. Bacterial meningitis in the United States in 1995. Active surveillance team. N Engl J Med. 1997;337:970-6. https://doi.org/10.1056/ NEJM199710023371404.

9. Thigpen MC, Whitney CG, Messonnier NE, Zell ER, Lynfield R, Hadler JL, et al. Bacterial meningitis in the United States, 1998-2007. N Engl J Med. 2011;364: 2016-25. https://doi.org/10.1056/NEJMoa1005384.

10. Brouwer MC, van de Beek D, Heckenberg SGB, Spanjaard L, de Gans J. Community-acquired Haemophilus influenzae meningitis in adults. Clin Microbiol Infect. 2007;13:439-42. https://doi.org/10.1111/j.1469-0691.2006.01670.x.

11. Center for Disease Control and Prevention (CDC). Haemophilus influenzae. In: Hamborsky J, Kroger A, Wolfe S, editors. Epidemiology and Prevention of Vaccine-Preventable Diseases. 13th ed., Washington D.C.: Public Health Foundation; 2015.

12. Center for Disease Control and Prevention (CDC). Pneumococcal Disease. In: Hamborsky J, Kroger A, Wolfe S, editors. Epidemiology and Prevention of Vaccine-Preventable Diseases. 13th ed., Washington D.C.: Public Health Foundation; 2015.

13. Johnson HL, Deloria-Knoll M, Levine OS, Stoszek SK, Freimanis Hance L, Reithinger $R$, et al. Systematic evaluation of serotypes causing invasive pneumococcal disease among children under five: the pneumococcal global serotype project. PLoS Med. 2010;7 https://doi.org/10.1371/journal. pmed.1000348.

14. Kalin M, Örtqvist Å, Almela M, Aufwerber E, Dwyer R, Henriques B, et al. Prospective study of prognostic factors in community-acquired Bacteremic pneumococcal disease in 5 countries. J Infect Dis. 2000;182:840-7. https://doi.org/10.1086/315760. 
15. Griffin MR, Zhu Y, Moore MR, Whitney CG, Grijalva CG. U.S. hospitalizations for pneumonia after a decade of pneumococcal vaccination. N Engl J Med. 2013;369:155-63. https://doi.org/10.1056/NEJMoa1209165.

16. Martinelli D, Pedalino B, Cappelli MG, Caputi G, Sallustio A, Fortunato F, et al. Towards the 13-valent pneumococcal conjugate universal vaccination. Hum Vaccin Immunother. 2014;10:33-9. https://doi.org/10.4161/hv.26650.

17. Baldo V, Cocchio S, Gallo T, Furlan P, Clagnan E, Del Zotto S, et al. Impact of pneumococcal conjugate vaccination: a retrospective study of hospitalization for pneumonia in north-East Italy. J Prev Med Hyg. 2016;57:E61-8.

18. Gladstone RA, Jefferies JM, Faust SN, Clarke SC. Pneumococcal 13-valent conjugate vaccine for the prevention of invasive pneumococcal disease in children and adults. Expert Rev Vaccines. 2012;11:889-902. https://doi.org/ 10.1586/erv.12.68

19. Halperin SA, Bettinger JA, Greenwood B, Harrison LH, Jelfs J, Ladhani SN, et al. The changing and dynamic epidemiology of meningococcal disease. Vaccine. 2012;30(Suppl 2):B26-36. https://doi.org/10.1016/j.vaccine.2011.12.032.

20. Trotter C, Samuelsson S, Perrocheau A, de Greeff S, de Melker H, Heuberger $S$, et al. Ascertainment of meningococcal disease in Europe. Euro Surveill. 2005;10:247-50.

21. Trotter $C L$, Ramsay ME. Vaccination against meningococcal disease in Europe: review and recommendations for the use of conjugate vaccines. FEMS Microbiol Rev. 2007;31:101-7. https://doi.org/10.1111/j.1574-6976.2006.00053.x.

22. National Center of Epidemiology and Surveillance and Health Promotion (CNESPS), National Institute for Health (Istituto Superiore di Sanità ISS). Data and evidences for the use of anti-pneumococcal vaccines in risk subjects of all ages and for the eventual vaccination of the elder population. Rome: 2013.

23. Italian Ministry of Health, National Institute for Health (Istituto Superiore di Sanità ISS). Italian Vaccine Action Plan 2016-2018. 2015

24. Gasparini R, Amicizia D, Lai PL, Panatto D. Meningococcal B vaccination strategies and their practical application in Italy. J Prev Med Hyg. 2015;56: e133-E139.

25. Watson PS, Turner DPJ. Clinical experience with the meningococcal B vaccine, Bexsero( $\left.{ }^{\oplus}\right)$ : prospects for reducing the burden of meningococcal serogroup B disease. Vaccine. 2016;34:875-80. https://doi.org/10.1016/j. vaccine.2015.11.057.

26. National Institute for Health (Istituto Superiore di Sanità ISS). Surveillance data on invasive bacterial diseases updated to April 4th 2016. Rome: 2016.

27. Stefanelli P, Miglietta A, Pezzotti P, Fazio C, Neri A, Vacca P, et al. Increased incidence of invasive meningococcal disease of serogroup C / clonal complex 11, Tuscany, Italy, 2015 to 2016. Euro Surveill. 2016;21:1-5. https://doi.org/10.2807/1560-7917.ES.2016.21.12.30176.

28. Istitution of Tuscany Region. Vaccination campaign against meningococcal C - Measures of prophylaxis and prevention 2016. http://www.regione. toscana.it/-/campagna-contro-il-meningococco-c.

29. Italian Ministry of Health. Circolare n. 5783 del $1^{\circ}$ marzo 2016. 2016.

30. Italian Ministry of Health. Decreto Ministeriale 28 Dicembre 1991. Istituzione della scheda di dimissione ospedaliera. 1992 G.U. 17 gennaio, n.13.

31. Italian National Statistical Institute (ISTAT). Geo-Demo ISTAT: maps, population and demographic statistics. http://demo.istat.it/index.html.

32. Italian Ministry of Health. Immunization in pediatric age: Vaccination coverage. http://www.salute.gov.it/portale/documentazione/p6_2_8_3_1. jsp?lingua=italiano\&id $=20$.

33. National Institute for Health (Istituto Superiore di Sanità ISS). ICONA 2003: National infant vaccination coverage in Italy. Rapporti ISTISAN 03/37 2003.

34. National Institute for Health (Istituto Superiore di Sanità ISS). ICONA 2008: National vaccination coverage survey among children and adolescents. Rapporti ISTISAN 09/29 2009.

35. Armitage P, Berry G, Matthews J. Statistical methods in medical research. 3rd ed. Oxford: Blackwell Scientific Publications; 1994

36. Kim HJ, Fay MP, Feuer EJ, Midthune DN. Permutation tests for joinpoint regression with applications to cancer rates. Stat Med. 2000;19:335-51.

37. Charlson ME, Pompei P, Ales KL, MacKenzie CR. A new method of classifying prognostic comorbidity in longitudinal studies: development and validation. J Chronic Dis. 1987;40:373-83.

38. Quan $H$, Sundararajan V, Halfon P, Fong A, Burnand B, Luthi J-C, et al. Coding algorithms for defining comorbidities in ICD-9-CM and ICD-10 administrative data. Med Care. 2005;43:1130-9.

39. de Waure C, Miglietta A, Nedovic D, Mereu G, Ricciardi W. Reduction in Neisseria meningitidis infection in Italy after meningococcal C conjugate vaccine introduction: a time trend analysis of 1994-2012 series. Hum Vaccin
Immunother. 2016;12:467-73. https://doi.org/10.1080/21645515.2015. 1078951.

40. Trotter $\mathrm{CL}$, Maiden MCJ. Meningococcal vaccines and herd immunity: lessons learned from serogroup C conjugate vaccination programmes. Expert Rev Vaccines. 2014;8:851-61. https://doi.org/10.1586/erv.09.48.

41. Trotter CL, McVernon J, Ramsay ME, Whitney CG, Mulholland EK, Goldblatt $D$, et al. Optimising the use of conjugate vaccines to prevent disease caused by Haemophilus influenzae type b, Neisseria meningitidis and Streptococcus pneumoniae. Vaccine. 2008;26:4434-45. https://doi.org/10. 1016/j.vaccine.2008.05.073.

42. Heymann D, Aylward B. Mass vaccination in public health. Control Commun. Dis. Man. 19th ed., Washington D.C.: American Public Health Association; 2008

43. Weil-Olivier C, Gaillat J. Can the success of pneumococcal conjugate vaccines for the prevention of pneumococcal diseases in children be extrapolated to adults? Vaccine. 2014;32:2022-6. https://doi.org/10.1016/j. vaccine.2014.02.008.

44. Kanerva M, Ollgren J, Virtanen MJ, Lyytikäinen O, Prevalence Survey Study Group. Risk factors for death in a cohort of patients with and without healthcare-associated infections in Finnish acute care hospitals. J Hosp Infect. 2008;70:353-60. https://doi.org/10.1016/j.jhin.2008.08.009.

45. Falsetti L, Viticchi G, Tarquinio N, Silvestrini M, Capeci W, Catozzo V, et al. Charlson comorbidity index as a predictor of in-hospital death in acute ischemic stroke among very old patients: a single-cohort perspective study. Neurol Sci. 2016;37:1443-8. https://doi.org/10.1007/s10072-016-2602-1.

46. Germinario C, Tafuri S, Vece MM, Prato R. Pneumococcal polysaccharide immunization strategies in Italian regions. Ig Sanita Pubbl. 2010;66:659-70.

47. Bonten MJM, Huijts SM, Bolkenbaas M, Webber C, Patterson S, Gault S, et al. Polysaccharide Conjugate Vaccine against Pneumococcal Pneumonia in Adults. N Engl J Med. 2015;372:1114-25. https://doi.org/10.1056/ NEJMoa1408544.

48. Ludwig E, Bonanni P, Rohde G, Sayiner A, Torres A. The remaining challenges of pneumococcal disease in adults. Eur Respir Rev. 2012;21.

49. Gibson GJ, Loddenkemper R, Lundbäck B, Sibille $Y$. Respiratory health and disease in Europe: the new European lung white book. Eur Respir J. 2013;42.

50. Daly E, Mason A, Goldacre M. Using case fatality rates as a health outcome indicator: literature review | National Centre for health outcomes development (NCHOD). 2000.

\section{Ready to submit your research? Choose BMC and benefit from:}

- fast, convenient online submission

- thorough peer review by experienced researchers in your field

- rapid publication on acceptance

- support for research data, including large and complex data types

- gold Open Access which fosters wider collaboration and increased citations

- maximum visibility for your research: over $100 \mathrm{M}$ website views per year

At BMC, research is always in progress.

Learn more biomedcentral.com/submissions 